\title{
Estenose na artéria renal: a necessidade de validação dos critérios diagnósticos no laboratório vascular
}

\author{
Renal artery stenosis: need for validation of diagnostic criteria in the vascular laboratory
}

\author{
Carlos Alberto Engelhom, Ana Luiza Engelhorn, M aria Fernanda C assou *
}

\section{Resumo}

O bjetivo: Validar os critérios diagnósticos da ultra-sonografia vascular com Doppler colorido na identificação das estenoses hemodinamicamente significativas nas artérias renais.

M étodos: Foram estudadas, prospectivamente, pela ultrasonografia vascular com D oppler colorido, 137 artérias renais de 69 pacientes adultos com suspeita de hipertensão arterial renovascular. O s resultados da avaliação pela ultra-sonografia vascular com D oppler colorido foram comparados, de maneira duplo-cega, à arteriografia, sendo definidos os valores de corte (curva RO C) da velocidadesistólica, velocidade diastólica e índice renal-aorta que melhor identificam as estenoses hemodinamicamente significativas, com angulação do D oppler de 60․

Resultados: 0 pico de velocidade sistólica superior a $250 \mathrm{~cm} / \mathrm{s}$ foi o parâmetro de melhor acurácia $(87,2 \%)$ na identificação das estenoses hemodinamicamente significativas, seguido do índice renalaorta superior a $3,2(85,6 \%)$ e do pico de velocidade diastólica superior a $48 \mathrm{~cm} / \mathrm{s}(70,4 \%)$.

C onclusões: 0 s parâmetros recomendados pela literatura devem ser validados em cada laboratório vascular. Para o equipamento de ultra-som utilizado e para a população incluída neste estudo, os critérios diagnósticos para estenoses hemodinamicamente significativas nas artérias renais devem ser reajustados para velocidade sistólica $>250$ $\mathrm{cm} / \mathrm{s}$ e índice renal-aorta $>3,2$, com angulação do D oppler de $60^{\circ}$

Palavras-chaves: hipertensão, artéria renal, ultra-som.

\begin{abstract}
O bjective: To validate the diagnostic criteria of color Doppler ultrasound in the identification of hemodynamically significant stenoses in renal arteries.

Methods: The color Doppler ultrasound was used to prospectively study 137 renal arteries of 69 adult patients suspected of having renovascular arterial hypertension. The results of the color D oppler ultrasound assessment were double-blindly compared to the arteriography. Cut-off values (ROC curve) were defined for systolic velocity, diastolic velocity and renal-aortic ratio, which better identify hemodynamically significant stenoses, at a D oppler angle of $60^{\circ}$.

Results: Peak systolic velocity higher than $250 \mathrm{~cm} / \mathrm{s}$ was the parameter for greater accuracy $(87.2 \%)$ in the identification of hemodynamically significant sten oses, followed by the renal-aortic ratio greater than $3.2(85.6 \%)$ and peak diastolic velocity higher than $48 \mathrm{~cm} / \mathrm{s}(70.4 \%)$.

Conclusions: Parameters recommended in the literature must be validated in every vascular laboratory. For the ultrasound device and population included in this study, the diagnostic criteria for hemodynamically significant stenoses of renal arteries must be readjusted to systolic velocity $>250 \mathrm{~cm} / \mathrm{s}$ and renal-aortic ratio > 3.2 , at a D oppler angle of 60 ․
\end{abstract}

Key words: hypertension, renal artery, ultrasonography.

eo IRA superior a 3,5 são osparâmetrosqueapresentam a melhor acurácia na identificação das estenoses potencialmente desencadeadoras da hipertensão renovascu$\operatorname{lar}^{1}$. 0 utrosautores, no entanto, preconizam valores de VS entre 100 e $200 \mathrm{~cm} / \mathrm{s}$ e IRA entre 3,2 e 3,5 como critériosdiagnósticosdasestenoseshemodinamicamente significativas ${ }^{2,3}$. N a presença de limitações técnicas na aval iação dos segmentos proximais das artérias renais, é possível estudar somente a região hilar da artéria pelo cálculo do índice e do tempo de aceleração, considerados parâmetros indiretos de avaliação ${ }^{4}$. 
O objetivo deste estudo foi determinar, em uma população aval iada por um equipamento específico, quais os parâmetros da UV D C que melhor identificam as estenoses hemodinamicamente significativas nas artérias renais, quando comparados à arteriografia.

\section{População e métodos}

Foi realizado, no Laboratório $\mathrm{V}$ ascular $\mathrm{N}$ ão Invasivo do H ospital de Caridade da Santa Casa de $M$ isericórdia de Curitiba - Pontifícia U niversidade C atólica do Paraná (PU CPR ), um estudo prospectivo, duplo-cego, para validar os critérios da UVDC no diagnóstico das estenoses hemodinamicamente significativas nas artérias renais, em rel ação à arteriografia, considerada o exame padrão-ouro.

\section{População}

Foram estudadas 137 artérias renais de 69 pacientes adultos, com média de idade de 54 anos, sendo 30 homense 39 mulheres, com suspeita de hipertensão arterial renovascular. U m dos pacientes estudados foi submetido à nefrectomia prévia ao estudo.

Foram incluídos pacientes jovens com hipertensão arterial de início recente; pacientes jovens sem história familiar de hipertensão; pacientes acima de 55 anos com hipertensão arterial de início recente ou descontrole abrupto; pacientes hipertensos refratários ao tratamento; pacientes hipertensos com sopro abdominal; e pacientes com hipertensão arterial predominantemente diastólica (acima de $120 \mathrm{mmH}$ g).

Foram excluídos os pacientes com diagnóstico prévio de lesão na artéria renal e histórico de alergia a contraste.

\section{Métodos}

Todos os pacientes aceitos no estudo foram submetidos ao exame ultra-sonográfico eao estudo arteriográfico, sem que os radiologistas e ultra-sonografistas vasculares tivessem conhecimento prévio do diagnóstico. O s exames ultra-sonográfico e arteriográfico do mesmo paciente foram interpretados, portanto, de maneira duplo-cega, segundo critérios de interpretação previamente definidos. 0 intervalo na realização dos exames ultra-sonográfico e arteriográfico foi inferior a 30 dias.

\section{Avaliação pela UVDC}

A avaliação anatômicaehemodinâmicapelaU VD C foi realizada pela técnica direta de visibilização das artérias renais, com os pacientes examinados em decúbito dorsal, com transdutores curvos de baixa freqüência (2 a 3,5 M H z) para permitir uma maior penetração (10-12 cm) do feixe de ultra-som.

Para a avaliação de fluxo, foi mantida uma caixa de cor pequena, com profundidade adequada para maximizar o número de quadros por minutos (frame rate) e a freqüência de repetição de pulsos (PRF) adequada para a estrutura a ser analisada. 0 ganho e o filtro da cor foram ajustados para permitir o mapeamento a cores do fluxo adequado.

O Doppler pulsado foi posicionado no centro do vaso nas artérias normais ou no local da alteração hemodinamicamente significativa, representada pelo aumento develocidades eturbilhonamento do fluxo. A amostra devolumefoi ajustada para o tamanho do vaso, evitando a contaminação com informação de outras estruturas vizinhas, principalmente devido aos movimentos respiratórios. Para a aorta abdominal e as artérias renais principais, foi mantido 0 ângulo de incidência do $D$ oppler pulsado o mais próximo de $60^{\circ}$. Foram ajustadoso ganho, filtro eescala das curvas de velocidade do Doppler pulsado, a fim de proporcionar uma curva adequada para a mensuração das velocidades.

A natomicamente, as artérias renais originam-se perpendicularmente do segmento proximal da aorta abdominal em direção à loja renal, em uma angulação próxima a $60^{\circ}$. Portanto, a origem e o segmento proximal das artérias renais foram avaliados longitudinalmente, por cortetransversal da aorta abdominal na região epigástrica. Por esse corte, na dependência da anatomia e biótipo do paciente, pôde-se, muitas vezes, avaliar quase toda a extensão das artérias renais. 0 s segmentos distais e o hilo renal, nas respectivas regiões lombares, foram avaliados por cortes longitudinais e transversais.

I dentificadas as artérias renais pel o mapeamento a cores do fluxo, em secção longitudinal, foi localizada a amostra de volume do D oppler pulsado para a obtenção da análise espectral e a mensuração das velocidades sistólica (VS) e diastólica (VD ), rotineiramente, na origem ou em qualquer outro segmento do vaso que apresentasse alterações de fluxo no mapeamento colorido, sempre com correção do ângulo de insonação do Doppler para $60^{\circ}$. 
A VS na aorta abdominal foi obtida, em corte longitudinal, no seu segmento proximal, ao nível da origem da artéria mesentérica superior, também respeitando um ângulo de insonação do $\mathrm{D}$ oppler de $60^{\circ}$ para efetuação do cálculo do IRA, isto é, a relação entrea VS das artérias renais e da aorta abdominal.

Para identificar as estenoses hemodinamicamente significativas, foram considerados os critérios da U niversidade de W ashington para valores absolutos da VS nas artérias renais e 0 IRA. As artérias renais foram consideradas normais com velocidades inferiores a $180 \mathrm{~cm} / \mathrm{sel}$ RA inferior a 3,5; com estenosesmoderadas $(<60 \%$ ) com velocidades superiores a $180 \mathrm{~cm} / \mathrm{s}$ e IRA inferior a 3,5; e estenoses hemodinamicamente significativas (>60\%) com velocidades superiores a $180 \mathrm{~cm} / \mathrm{s}$ eIRA superior a $3,5^{1}$.

Preferencialmente, os exames foram realizados no período da manhã, e os pacientes foram orientados a permanecer em jejum nas 12 horas antecedentes ao exame.

\section{Avaliação arteriográfica}

A avaliação anatômica das obstruções arteriais pela imagem arteriográfica foi realizada pela técnica de subtração digital, por cateterismo arterial sel etivo, via punção da artéria femoral, estudando-se as artérias renais e do parênquima renal, assim como a excreção do agente de contraste.

A mensuração do grau de estenose na artéria renal foi obtida pela relação entre o diâmetro da artéria normal eo segmento comprometido do vaso. Asartérias renais normais apresentaram-se contrastadas em toda a extensão, com paredes regulares, incluindo a visibilização das artérias intraparenquimatosas. As estenoses foram quantificadas em: moderadas, inferiores a $60 \%$ de redução anatômica do diâmetro da luz arterial; e significativas, superiores a $60 \%$ de redução anatômica do diâmetro da luz arterial, com diminuição da excreção renal do contraste. $\mathrm{N}$ as obstruções, não foi contrastada a artéria renal na sua topografia habitual eausência de contraste no parênquima renal.

\section{Equipamentos}

Para avaliação arterial ultra-sonográfica, foi utilizado o equipamento Siemes-Sonoline Elegra ${ }^{\circledR}$. Para a avaliação arteriográfica, foi utilizado o aparelho com subtração digital de imagens Siemens Angioscopy ${ }^{\circledR}$.

\section{Análise estatística}

Para a diferenciação das estenoses hemodinamicamentesignificativas $(>60 \%$ ) das estenosesmoderadase das artérias normais pel os critérios diagnósticos anteriormente citados (VS > $180 \mathrm{~cm} / \mathrm{s}$ e IRA $>3,65$ ), foram calculados osíndices de qualidade (sensibilidade, especificidade, acurácia, valor preditivo positivo e valor preditivo negativo).

$\mathrm{N}$ a determinação da sensibilidade e especificidade dos valores de VS, VD e IRA na detecção de estenose hemodinamicamente significativa ( $>60 \%$ de redução do diâmetro da luz do vaso), foi considerada a curva RO C para cada parâmetro individual e comparativamente entre os três parâmetros.

\section{Resultados}

Baseada noscritérios deVS $>180 \mathrm{~cm} /$ sel RA $>3,5$, a comparação entre os métodos, excluindo sete artérias inconclusivas, revelou que, das 130 artérias restantes, 116 artérias tiveram laudos concordantes (89,2\%) e 14 discordantes $(10,8 \%)$.

D as 116 artérias com laudos concordantes, 40 foram consideradas normais, seis com estenoses moderadas, 63 com estenoseshemodinamicamentesignificativas e sete obstruídas.

D as 14 artérias renais com laudos discordantes, nove apresentaram resultados falso-positivos e cinco falso-negativos da UVDC em relação à arteriografia. Em todas as nove artérias consideradas como resultado falso-positivo, a VS detectada foi superior a $200 \mathrm{~cm} / \mathrm{s}$, e em cinco delas, o IRA foi superior a 4. A artérias consideradas como resultado fal so-negativo apresentaram IRA inferioresa 3,5, com VS próximasa $200 \mathrm{~cm} / \mathrm{s}$.

O s valores de sensibilidade, especificidade, valor preditivo positivo evalor preditivo negativo na diferenciação da estenose hemodinamicamente significativa (>60\%) das estenoses moderadas e das artériasnormais foram respectivamente $92,6 \%, 83,6 \%, 87,5 \%$ e $90 \%$. A acurácia geral daUVD C em relação à arteriografia foi de $91,94 \%$

Em relação aos parâmetros diagnósticos de velocidade de pico sistólico, de velocidade de pico diastólico e de IRA, a análise estatística revel ou o seguinte:

a) Velocidadesistólica - A velocidade de $252 \mathrm{~cm} / \mathrm{sfoi}$ 0 ponto de corte estimado. Para essa vel ocidade, a sensibilidade estimada foi de $83,33 \%$. 0 intervalo de $95 \%$ de confiança para a sensibilidade é de 
$72,05-91,43 \%$. Já em relação à especificidade, 0 valor estimado foi igual a $91,52 \%$, com intervalo de 95\% de confiança igual a 81,19-97,27\% . A acurácia calculada, com basenos dados, para aV S de 252 foi igual a 87,2\%. A área estimada sob a curva RO C (Figura 1) para VS foi igual a $92,1 \%$.

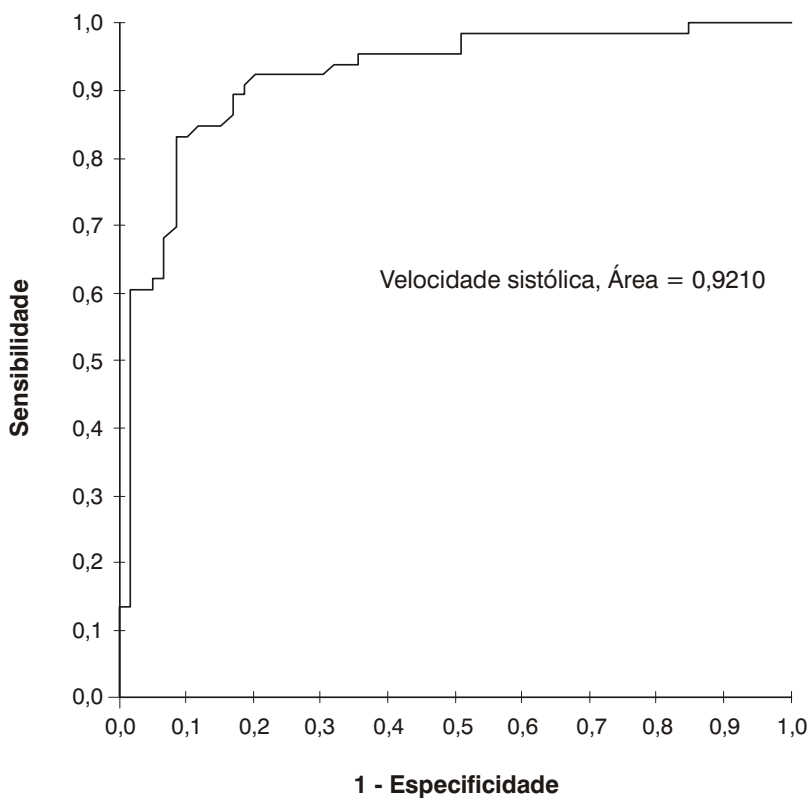

Figura 1 - C urva RO C para a velocidade de pico sistólico

b) Velocidadediastólica - A velocidade de $48 \mathrm{~cm} / \mathrm{sfoi}$ o ponto de corte estimado. Para essa velocidade, a sensibilidadeestimadafoi de $69,7 \%$. 0 intervalo de $95 \%$ de confiança para a sensibilidade é de 57,09$80,45 \%$. Já em relação à especificidade, 0 valor estimado foi igual a $71,19 \%$, com intervalo de $95 \%$ de confiança igual a 57,86-82,28\%. A acurácia calculada, com basenos dados, para aVD de 48 foi igual a 70,4\%. A área estimada sob a curva RO C (Figura 2) para VD foi igual a 76,98\%.

c) Índicerenal-aorta - 0 IRA de 3,27 foi o ponto de corte estimado. Para esse índice, a sensibilidade estimada foi de $84,85 \%$. 0 intervalo de $95 \%$ de confiança para a sensibilidadeé de 73,81-92,54\%. Já em relação à especificidade, o valor estimado foi igual a $86,44 \%$, com intervalo de $95 \%$ deconfiança

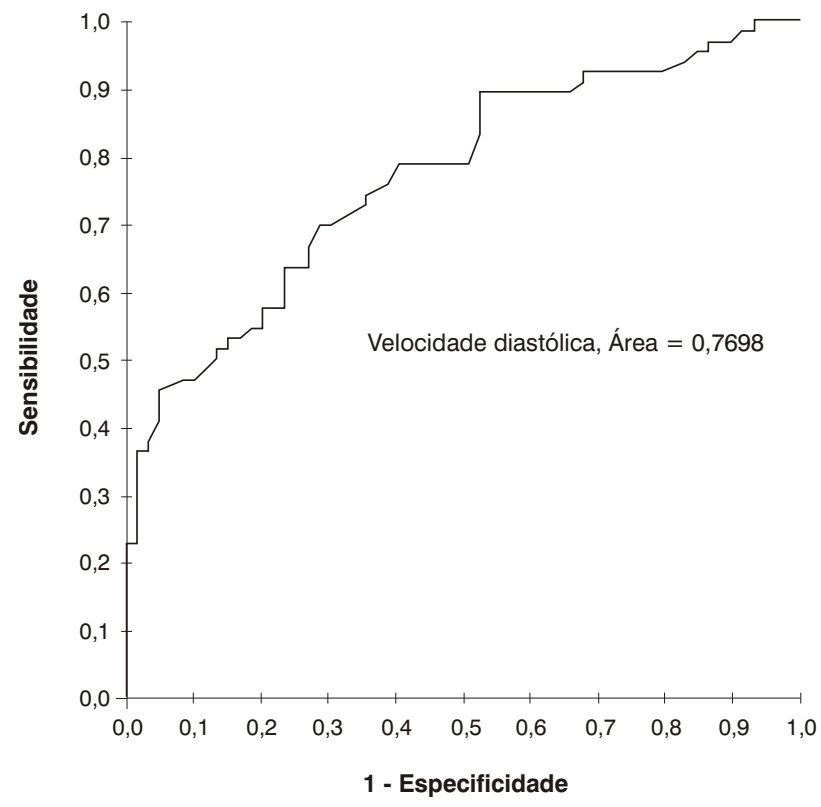

Figura 2 - Curva ROC para a velocidade de pico diastóliCO

igual a 74,92-94,03\%. A acurácia calculada, com base nos dados, para o IRA de 3,27 foi igual a 85,6\%. A área estimada sob a curva R O C (Figura3) para VD foi igual a $89,87 \%$.

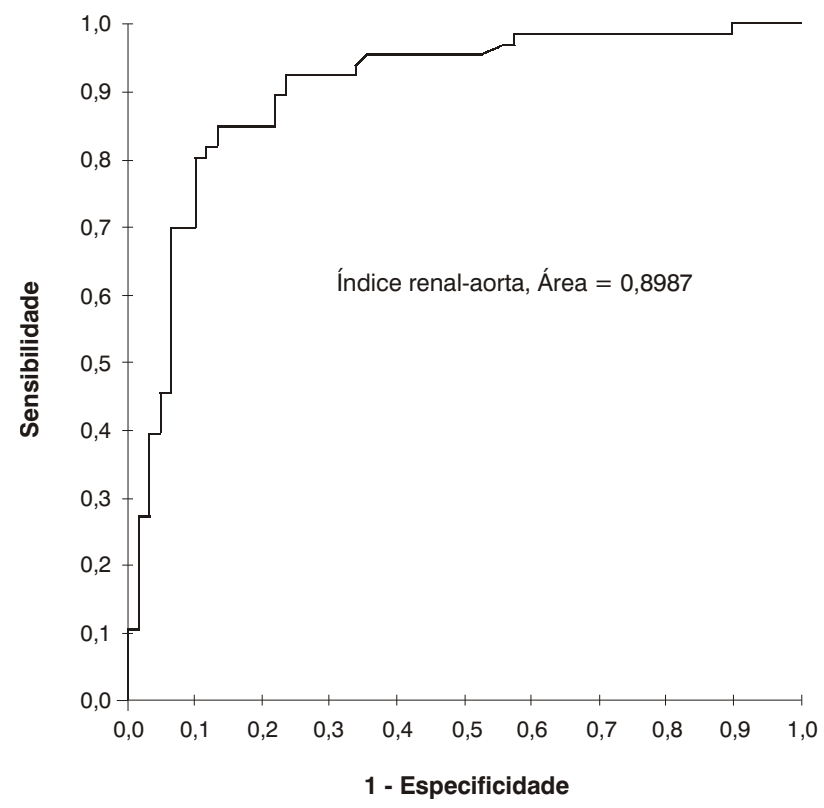

Figura 3 - Curva ROC para o índice renal-aorta 
d) Comparação entre os parâmetros - 0 resultado deste estudo indicou que a área sob a curva ROC (Figura 4) para a VS foi a maior entre os três parâmetros (92,1\%). A segunda maior área sob a curva ROC foi a do IRA $(89,87 \%)$. 0 parâmetro que apresentou a menor área foi a VD (76,98\%). D esta forma, esses resultados indicam quea VS éo melhor parâmetro para corretamente classificar os pacientes entre normal ou estenose moderada e estenose hemodinamicamente significativa.

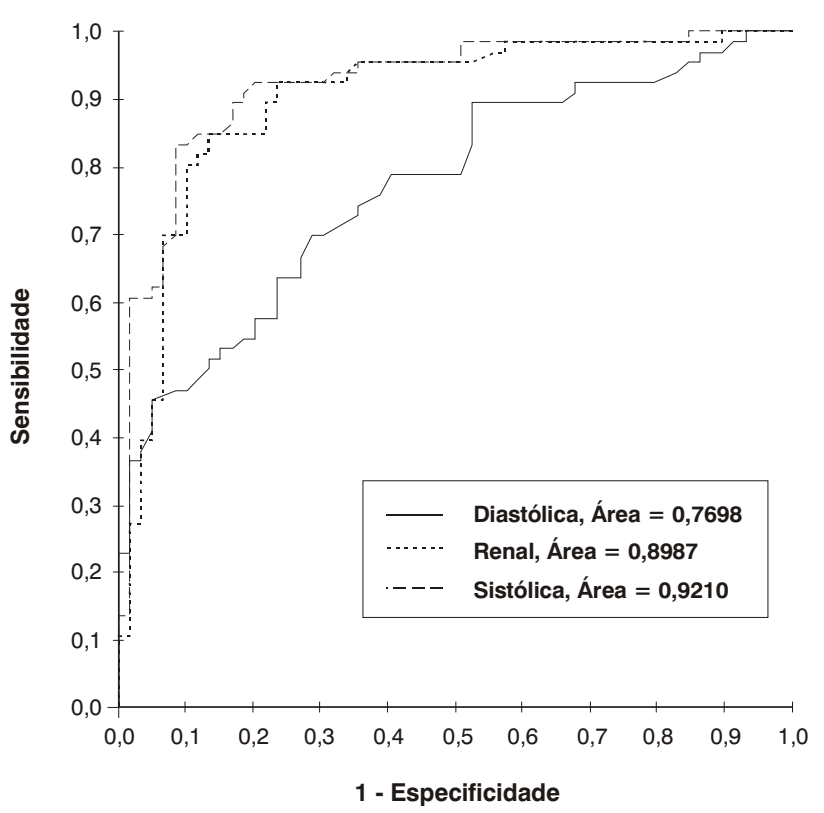

Figura 4 - C C urva ROC comparativa entre os parâmetros estudados

\section{D iscussão}

A utilização da UVD C no diagnóstico das doenças vasculares tem sido ampliada nas últimas décadas, com a melhoria na sensibilidade do equipamento e com a utilização do mapeamento a cores do fluxo. 0 scritérios diretos da UVDC para o diagnóstico da estenose na artéria renal são baseadosna visibilização detoda extensão das artérias renais, na mensuração da VS e da VD e no IRA.

O valor diagnóstico da VS, proposto por vários autores, tem sido entre 100 a $200 \mathrm{~cm} / \mathrm{s}$. H ansen et al. consideram o valor de $180 \mathrm{~cm} / \mathrm{s}$ como base das alterações hemodinâmicas nas artérias renais ${ }^{5}$. M irales et al. consideram a velocidade de $198 \mathrm{~cm} / \mathrm{s}$. O llin et al. e Krumme et al. apontam a velocidade de $200 \mathrm{~cm} / \mathrm{s}^{6-8}$.

$D$ e acordo com os trabalhos iniciais da U niversidade deW ashington, o IRA maior ou igual a 3,5, adotado por vários autores, indica estenose de artéria renal hemodinamicamente significativa ${ }^{9-11}$. M irales et al. propõem um valor acima de 3,3 para esse critério, porém com valores de sensibilidade e especificidade semelhantes aos do grupo de W ashington ${ }^{6}$.

Considerando, neste trabalho, a análise individual dos parâmetros de VS, VD e IRA, os valores que apresentaram os melhores índices de acurácia foram, respectivamente, a velocidadedepico sistólico $(87,2 \%)$, olRA (85,6\%) eavelocidadedepico diastólico $(70,4 \%)$. D essesval ores, a vel ocidade de pico sistólico apresentou osmelhoresíndices de sensibilidade (83,33\% ) eespecificidade $(91,52 \%)$.

$M$ irales et al., em um estudo semelhante, definiram também a VS como o parâmetro de melhor acurácia na detecção de estenose hemodinamicamente significativa, mas no val or de corte de $198 \mathrm{~cm} / \mathrm{s}$, também seguido do IRA de $3,3^{6}$.

Em relação à $V S$, considerada o melhor parâmetro de discriminação das estenoses hemodinamicamente significativas, o val or devel ocidadesuperior a $252 \mathrm{~cm} / \mathrm{s}$, neste trabalho, foi determinado como o de maior acurácia. N o entanto, os valores encontrados na literatura sugerem a VS superior a $200 \mathrm{~cm} / \mathrm{s}$ como referência para as estenoses hemodinamicamente significativas. A explicação para essa diferença pode estar relacionada às características do equipamento utilizado ou ao critério técnico, adotado neste trabal ho, da escol ha de angulação fixa de $60^{\circ}$ para o D oppler pulsado na mensuração dasvelocidades, diferentementedamaioria dosautores, que utilizaram ângulos corrigidos inferiores a 60²,7-9.

Por isso, devido à possibilidade de variação nas medidas dos diferentes equipamentos de UVDC, os parâmetros recomendados pela literatura devem ser validados em cada laboratório vascular. Para o equipamento de ultra-som utilizado neste estudo, os critérios diagnósticos devem ser reajustados para VS $>250 \mathrm{~cm} / \mathrm{s}$ e IRA >3,2, para uma melhor acurácia na detecção das estenoses $>60 \%$ nas artérias renais.

\section{R eferências}

1. Strandness $\mathrm{rDE}$. D uplexscanning in diagnosis of renovascular hypertension. Surg Clin N orth Am. 1990;70:109-17. 
2. D esberg A, Pausther D M , Lammert GK, et al. Renal artery stenosis: evaluation with color Doppler flow imaging. Radiology. 1990;12:227-36.

3. Berland LL, Koslin DB, Routh WD, Keller FS. Renal artery stenosis: prospectiveevaluation of diagnosis with color duplex ultrasound compared with angiography. Radiology. 1990;174:421-3.

4. N azzal M M, Hoballah JJ, M iller EV, et al. Renal hilar $D$ oppler analysis is of value in the management of patients with renovascular disease. Am J Surg. 1997;174:164-8.

5. H ansen KJ, Tribble RW, Reavis SW, et al. Renal D uplex sonography: evaluation of clinical utility. J Vasc Surg. 1990;12:250-7.

6. M irales M , C airols M , C otillas], G imenezA, Santiso A. V alue of $D$ oppler parametersin thediagnosis of renal artery stenosis. J V asc Surg. 1996;23:428-35.

7. Ollin JW, Piedmonte M R, Yoing JR, et al. The utility of duplex ultrasound scanning of therenal arteriesfor diagnosing significant renal artery stenosis. Ann Intern Med. 1995;122:833-8.
8. Krumme $B$, Blum $U$, Schwertfeger $E$, et al. Diagnosis of renovascular diseaseby intraand extrarenal $D$ oppler scanning. Kidney Int. 1996;50:1288-92.

9. Kohler T R, Zieler, M artin RL, et al. $\mathrm{N}$ on invasivediagnosis of renal artery by ultrasonic duplex scanning. J Vasc Surg. 1986;4:450-6.

10. H offmann U, Edwards JM, C arter S, et al. Role of duplex scanning for the detection of aterosclerotic renal artery disease. Kidney Int. 1991;39:1232-9.

11. T aylor DC, Kettler M D, M oneta G L, et al. Follow-up of renal artery stenosisby duplexultrasound. J V asc Surg. 1988;7:633-9.

Correspondência:

Carlos Alberto Engelhorn

Rua D eputado H eitor Alencar Furtado, 1720/901

CEP: 81200-110 - Curitiba, PR

Tel.: (41) 279.1241

E-mail: engelhor@bsi.com.br 